\title{
A Multi-Functional De-Icing Equipment Using Hybrid Type Parallel Current Source Converters
}

\author{
Yang Hu ${ }^{1}$, Heng Zhang ${ }^{1}$, Jing Chen ${ }^{1}$, Huan Zhang ${ }^{1}$, Xinmeng Liu ${ }^{1}$, Jiachen Li $^{1}$, Hanyu Chen ${ }^{1}$, Haiyang Hu ${ }^{1}$ \\ ${ }^{1}$ State Grid Corporation of China, China
}

\begin{abstract}
This paper has proposed a phase controlled and PWM controlled power current source converter (CSC) for transmission line de-icing. The proposed system consists of a high-power thyristor-based line commutated converter to contribute a majority of the de de-icing current and a low power PWM CSC to provide a part of the de de-icing current and to compensate the low order harmonic ac current from the TCR. Thus, the grid current can be highly sinusoidal. In order to avoid the resonance caused by the interaction between TCR and PWM CSC, a virtual impedance control is implemented in the PWM CSC without using any additional sensors. Comprehensive verification results have been obtained to validate the correctness of the proposed approach.
\end{abstract}

\section{Introduction}

Heavy icing on transmission lines can cause serious damages to modern power system equipment such as the line broken and the collapses of tower, which may even lead to further serious consequences including power system blackout. These accidents have been reported in recent years [1]-[3].

To ensure the secure operation of transmission lines and towers, active de-icing through using power electronics equipment has gained growing interest in recent years. There are two types of de-icing approaches, namely ac de-icing and dc de-icing, according to the type of the current flowing through the transmission line. When ac current is applied, it is nontrivial amount of reactive power flow on the line, which is a huge loss regarding power converter capacity. Alternatively, dc deicing has been considered as a good candidate due to its simple control and there is no involvement of reactive power flow.

Moreover, in recent years, the modular multi-level voltage source converter has been considered as an alternative candidate. Comparing to the line commutated converters, a PWM voltage source can accurate control the ac line current with minimized distortion [5]. However, as VSC based rectifier only controls the dc voltage instead of the dc current, the accuracy of de-icing current regulation can be affected. In more recent years, the modular multi-level converter has been studied to dc de-icing application. Comparing to the conventional twolevel voltage source converter, MMC system features modular configuration that is easy for maintenance and capacity expansion. In addition, the possibility of using de-icing equipment for auxiliary services including reactive power compensation has been discussed.
However, it is necessary to point out that MMC system is typically bulky and cost expensive and the control of MMC including de voltage balancing is complicated.

On the other hand, it is seen that the PWM current source converter (CSC) has been widely used in high power applications including medium voltage variable speed drives and energy storage using superconductive coils. As the dc current of CSC is fully controlled, it is considered as a good candidate for dc de-icing. However, the capacity of a single CSC is limited due to the limitation of withstand voltage and current of power electronics devices. To enhance to power transfer capacity, using parallel CSC modules to construct a highpower system is study. In [6], a direct parallel connection of CSC modules is proposed and the selection of space vectors is discussed in order to obtain proper dc rail current balancing. Furthermore, to reduce the cost on the common mode choke or alleviate the stress of isolation transformers, the common mode voltage reduction of parallel CSC modules is also discussed. It has been validated that the parallel CSC can achieve simultaneous dc rail current balancing and common mode voltage reduction via the proper selection of redundant space vector switching state. However, it is necessary to note that using fully PWM CSC to obtain controllable dc output current is cost expensive.

To deal with the aforementioned limitation, this paper has proposed a hybrid parallel converter module-based dc de-icing system, where a high power but low cost LCC converter is adopted to provide the majority of the dc current while a low power PWM CSC is adopted to provide an accurate adjustment of the dc current and to compensate the ac side harmonic component from the LCC converter. It has been validated that by using an internal virtual impedance control in the PWM CSC, the risk of interactive resonance between parallel converter

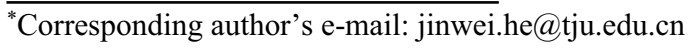


module output filters can be mitigated, and there is no need to increase any measurement in the process of implementing the virtual impedance. Comprehensive simulation results have been provided.

\section{The proposed system}

This paper starts with the introduction to the configuration of the proposed system with the focus on the cost reduction using LCC converter. Then, a detailed introduction to the control of $\mathrm{dc}$ rail current and compensation of ac line current low order harmonic component.

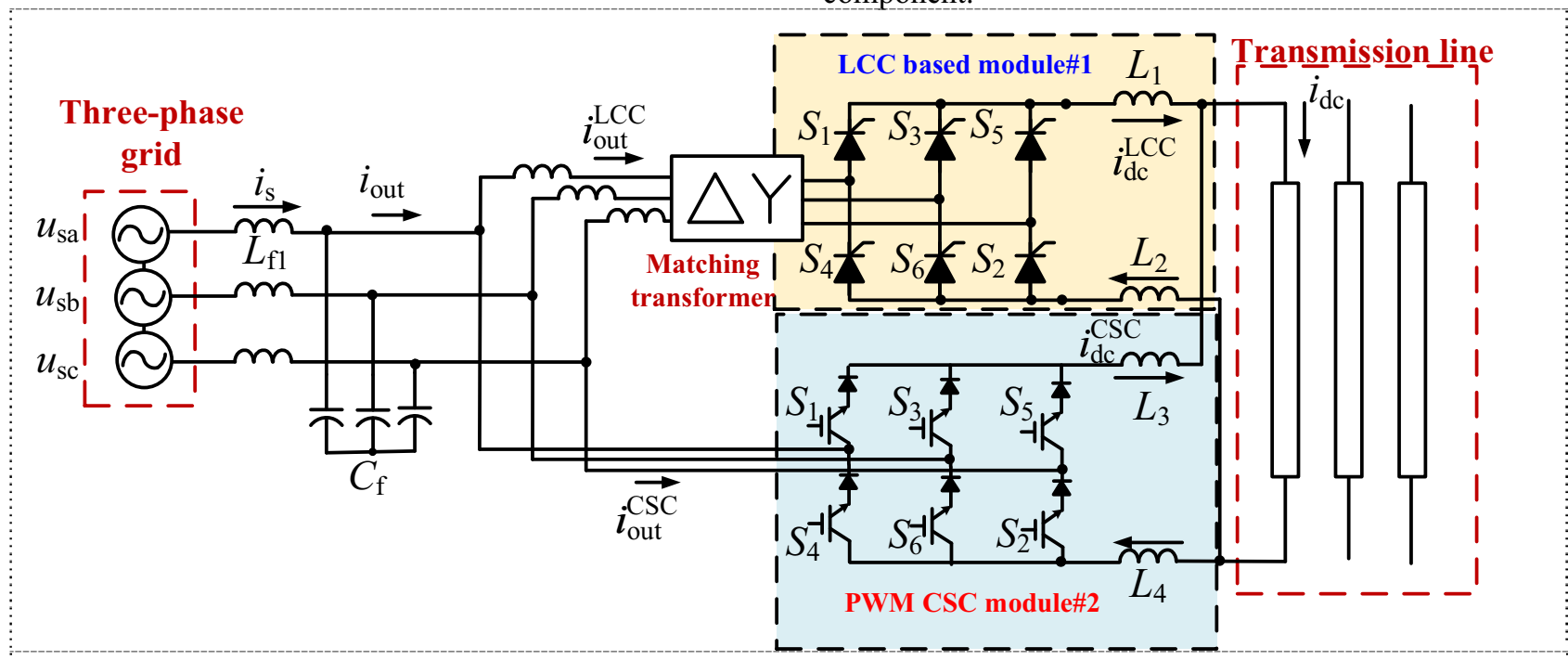

Figure 1. Circuit diagram of the proposed hybrid current source based dc de-icing system.

\subsection{Circuit Configuration}

Fig. 1 shows the circuit diagram of the proposed hybrid current source system. Firstly, a low voltage three-phase grid is connected to proposed dc de-icing via an LC filter, where $u_{\mathrm{sa}}, u_{\mathrm{sb}}$, and $u_{\mathrm{sc}}$ are the instantaneous grid voltage and $C_{\mathrm{f}}$ and $L_{\mathrm{f}}$ are the LC filter capacitance and inductance, respectively. In the upper part of the system, the LCC converter is interfaced to the grid with a matching transformer. The LCC converter consists of six thryistor devices $S_{1}$ to $S_{6}$ and the dc terminals are connected to high power transmission line with dc chokes. The LCC converter output current is described as $i_{\text {out }}^{\mathrm{LCC}}$ and the dc current of LCC converter is $i_{\mathrm{dc}}^{\mathrm{LCC}}$. In order to reduce the cost of the dc de-icing system, the LCC converter is responsible for the majority of the power flow regulation.

In the lower part of the figure, a PWM CSC converter is placed in parallel with the LCC converter. Similarly, the PWM CSC converter consists of six devices as $S_{1}$ to $S_{6}$. The power device is constructed by using an IGBT device with a series connected diode in order to obtain reverse block capability. The output current of the PWM CSC converter is also connected to the transmission line via the dc chokes as $L_{3}$ and $L_{4}$. As mentioned earlier, the power capacity of the PWM CSC converter is typically lower than the LCC converter counterpart in order to reduce the cost of the entire dc de-icing system.

For this system, the high dc current for de-icing is controlled by the coordination operation of both converters. In addition, it is well understood that the LCC converter has nontrivial ripple current in the ac side, which is usually compensated by the tuned harmonic filters. For this system, the flexible control of PWM CSC also offers the possibility to actively compensate the ripples from LCC converter. Accordingly, the cost of the passive filters can be reduced. 


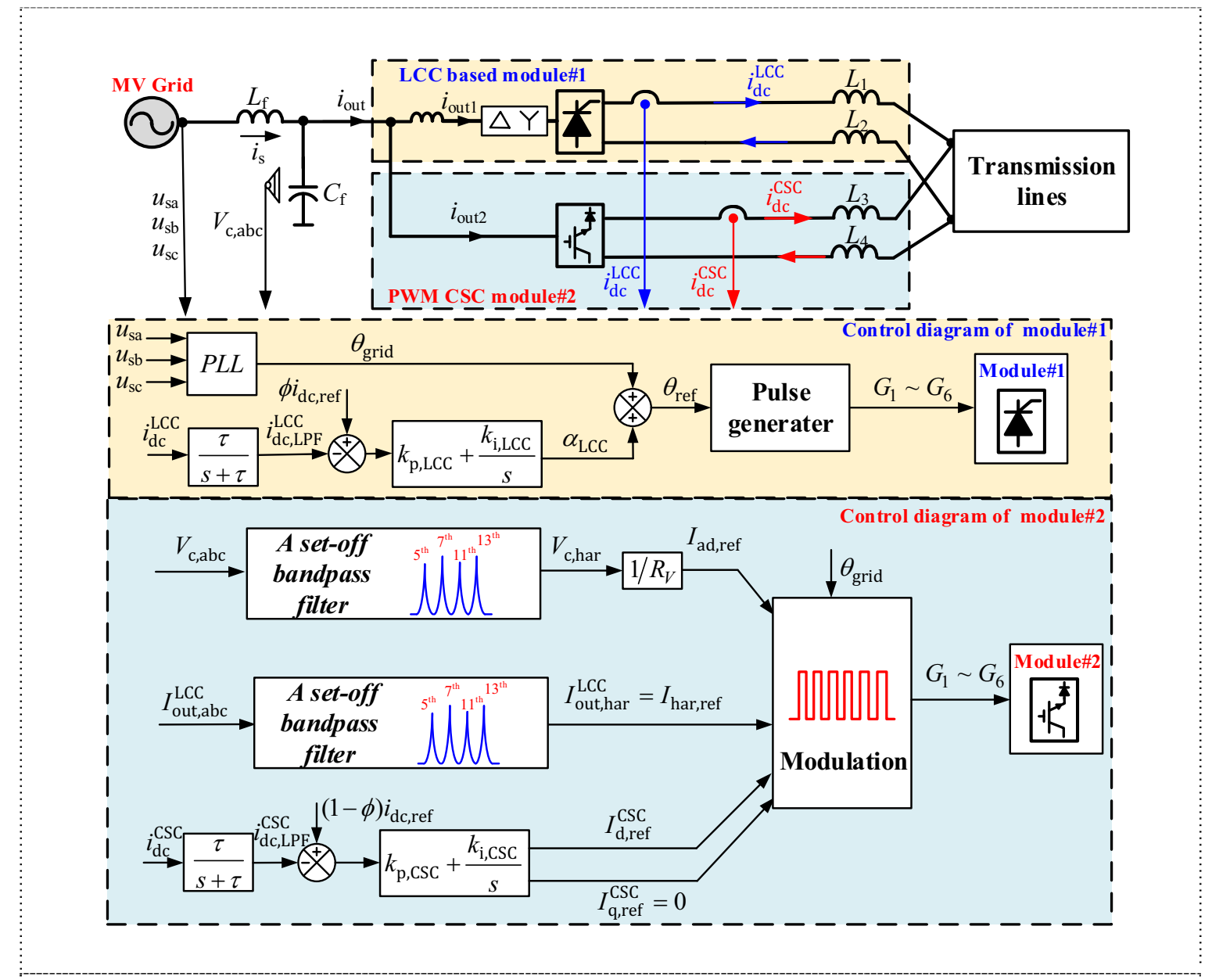

Figure 2. Control diagram of the proposed hybrid current source-based dc de-icing system.

\subsection{Controller Design}

In this subsection, the controller of this system is introduced in detail, with the focus on the dc current regulation, the compensation of the LCC converter ac ripples and the active damping of LC filter resonance. The control diagram of the proposed system is shown in Fig. 2.

Firstly, the dc current of the de-icing system is regulated by a hybrid controller as:

$$
\begin{gathered}
\alpha_{\mathrm{LCC}}=\left(k_{\mathrm{p}, \mathrm{LCC}}+\frac{k_{\mathrm{i}, \mathrm{LCC}}}{s}\right) \cdot\left(\phi i_{\mathrm{dc}, \mathrm{ref}}-i_{\mathrm{dc}, \mathrm{LPF}}^{\mathrm{LCC}}\right) \\
i_{\mathrm{dc}, \mathrm{LPF}}^{\mathrm{LCC}}=\frac{\tau}{s+\tau} i_{\mathrm{dc}}^{\mathrm{LCC}}
\end{gathered}
$$

where $i_{\mathrm{dc}}^{\mathrm{LCC}}$ is measured dc current, $i_{\mathrm{dc}, \mathrm{LPF}}^{\mathrm{LCC}}$ is the measured dc current after a low-pass filtering, $i_{\mathrm{dc}, \text { ref }}$ is the reference current for de-icing, $\phi$ is the weighting factor that is determined by the power rating of LCC converter and PWM CSC converter, $k_{\mathrm{p}, \mathrm{LCC}}$ is the proportional gain of the dc current regulator and $k_{\mathrm{i}, \mathrm{LCC}}$ is the integral gain of the regulator. The output of this is the firing angle $\alpha$ of LCC converter.

Similarly, the PWM CSC converter is also responsible for a part of the dc de-icing current. In this case, another dc current regulator is adopted for the PWM CSC converter as:

$$
\begin{gathered}
i_{\mathrm{d}, \mathrm{ref}}^{\mathrm{CSC}}=\left(k_{\mathrm{p}, \mathrm{CSC}}+\frac{k_{\mathrm{i}, \mathrm{CSC}}}{s}\right) \cdot\left((1-\phi) \cdot i_{\mathrm{dc}, \mathrm{ref}}-i_{\mathrm{dc}, \mathrm{LPF}}^{\mathrm{CSC}}\right) \\
i_{\mathrm{dc}, \mathrm{LPF}}^{\mathrm{CSC}}=\frac{\tau}{s+\tau} i_{\mathrm{dc}}^{\mathrm{CSC}}
\end{gathered}
$$

where $i_{\mathrm{dc} \text {, ref }}$ is the reference current, $i_{\mathrm{dc}, \mathrm{LPF}}^{\mathrm{CSC}}$ is the measured dc current of the PWM CSC converter with a low pass filter, the cut-off frequency of the low pass filter is $1 / \tau, k_{\mathrm{p}, \mathrm{CSC}}$ and $k_{\mathrm{i}, \mathrm{CSC}}$ are the proportional and integral gain of the regulator, the output of the regulator is the $\mathrm{d}$-axis reference current in synchronous rotating reference frame and the reference value of q-axis is zero. To synchronous the controller with the grid, the LC filter capacitor voltage is also measured and the phase angle $\theta_{\text {grid }}$ is determined by using a phase-locked loop (PLL).

It is necessary to note that the PWM CSC converter is also responsible for the compensation of the harmonic current from the LCC converter. This task can be realized by two steps. Firstly, the output current of the LCC converter is measured and its harmonic component is selectively extracted by using a set-off bandpass filter as: 


$$
\begin{aligned}
& I_{\text {out }, \text { har }}^{\mathrm{LCC}}=G_{\mathrm{har}, \mathrm{comp}}(s) \cdot I_{\text {out,abc }}^{\mathrm{LCC}} \\
& =\sum_{h=5,7,11,13} \frac{2 \omega_{\mathrm{c}} s}{s^{2}+2 \omega_{\mathrm{c}} s+\left(h \cdot \omega_{0}\right)^{2}} \cdot I_{\text {out,abc }}^{\mathrm{LCC}}
\end{aligned}
$$

where $h$ is the harmonic order, $\omega_{\mathrm{c}}$ is the bandpass angular frequency, $\omega_{0}$ is the nominal fundamental angular frequency. and $I_{\text {out, har }}^{\mathrm{LCC}}$ is the extracted LCC converter harmonic current. Then, the harmonic current reference can be obtained as $I_{\text {har, ref }}=I_{\text {out, har }}^{\mathrm{LCC}}$.

In addition, it is necessary to note that the output LC filter may have significant resonance caused by either the harmonic pollution from the grid voltage or from the LCC converter. To actively damp out the resonance but without using any additional measurement of voltage or current, this paper has adopted the concept of virtual impedance that is connected in parallel with the LC filter capacitor. The detailed control law is given below as:

$$
\begin{aligned}
& V_{\mathrm{c}, \mathrm{har}}=G_{\text {har,ad }}(s) \cdot V_{\mathrm{c}, \mathrm{abc}} \\
& =\sum_{h=5,7,11,13} \frac{2 \omega_{\mathrm{c}} s}{s^{2}+2 \omega_{\mathrm{c}} s+\left(h \cdot \omega_{0}\right)^{2}} \cdot V_{\mathrm{c}, \mathrm{abc}}
\end{aligned}
$$

where $h$ is the harmonic order, $\omega_{\mathrm{c}}$ is the bandpass angular frequency, $\omega_{0}$ is the nominal fundamental angular frequency. and $V_{\mathrm{c} \text {, har }}$ is the extracted filter capacitor harmonic voltage.

To realize the virtual damping resistor, a digital implementation scheme is developed as shown in Fig. 2. In Fig. 2 the fundamental and the harmonic capacitor voltage are first separated using a harmonic detector mentioned above. For the virtual damping resistance only effective at the harmonic frequencies, the associated harmonic damping current can be described as:

$$
I_{\text {ad,ref }}=\frac{V_{\mathrm{c}, \mathrm{har}}}{R_{\mathrm{V}}}=\frac{G_{\mathrm{har}, \mathrm{ad}}(s) \cdot V_{\mathrm{c}, \mathrm{abc}}}{R_{\mathrm{V}}}
$$

where $R_{\mathrm{V}}$ is the resistance of the virtual damping resistor, $G_{\text {har, ad }}(\mathrm{s})$ is the transfer function of the harmonic detector, which is constructed based on a set-off bandpass filter mentioned above.

When both the real power current component and reactive power component are determined, combined with the two harmonic components obtained above, the reference current can be obtained as:

$$
I_{\text {ref }}=\sqrt{\left(I_{\mathrm{d}, \text { ref }}^{\text {CSC }}\right)^{2}+\left(I_{\mathrm{q}, \text { ref }}^{\text {CSC }}\right)^{2}}+I_{\text {ad,ref }}-I_{\text {har, ref }}
$$

\section{Verification results}

In order to validate the effectiveness of the proposed CSC based de-icing equipment, relevant simulation has been conducted and the key results are presented. Similar to the diagram in Fig. 1, the simulated system consists of two power modules. Among them, an LCC converter is interfaced to the grid with isolation matching transformer, which is used to provide a large part of the dc rail current for de-icing. In addition, a CSC converter at lower power rating is used to further tune the dc rail current for an accurate dc de-icing and the ac side harmonic current from LCC converter can also be compensated at the same time.

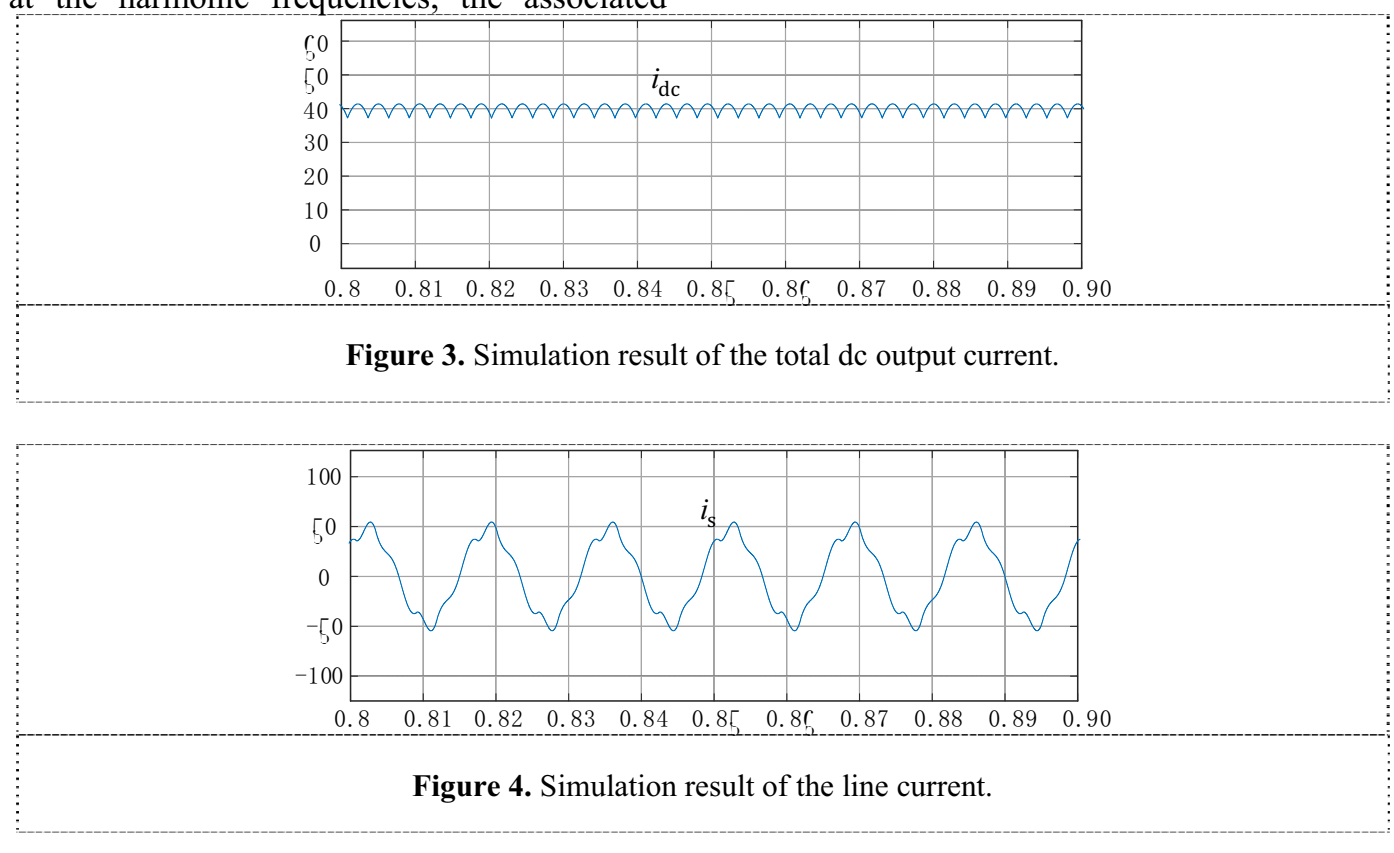




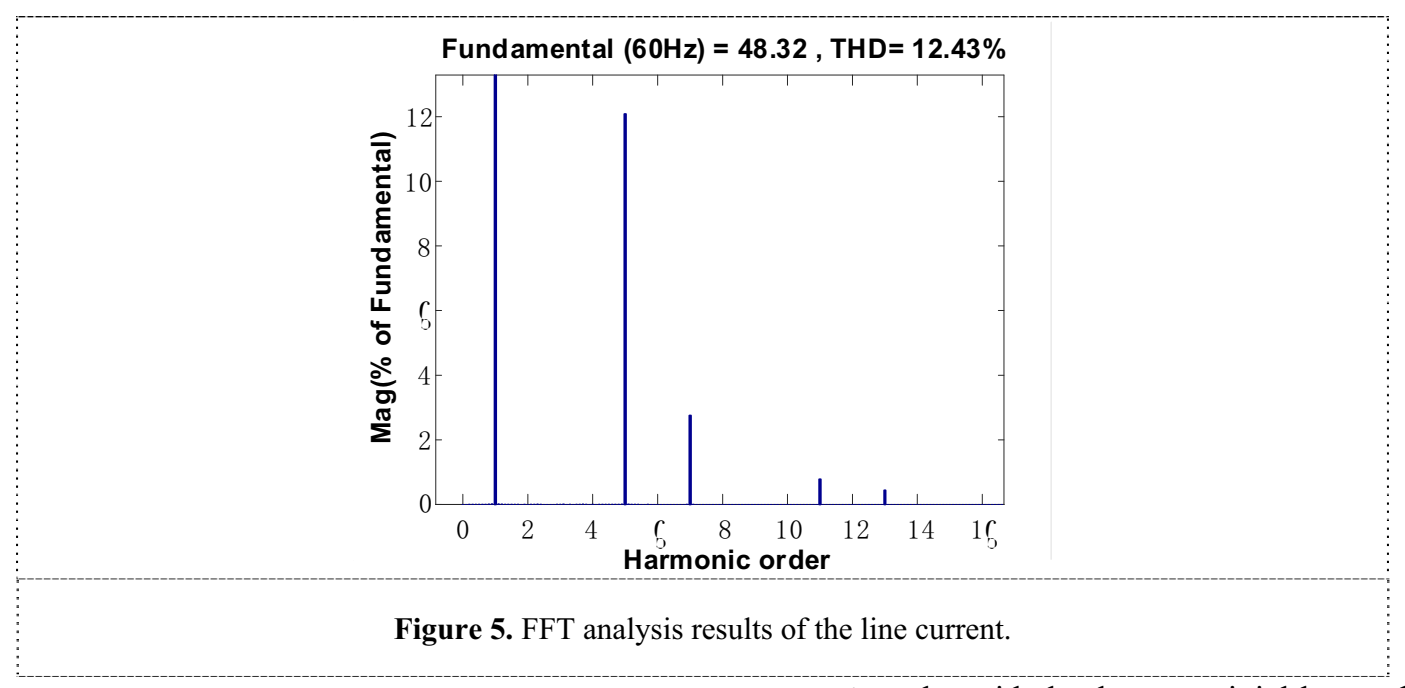

Firstly, the performance without using the CSC converter is obtained as shown in Fig. 3 and Fig. 4. It is shown that always a high dc current can be obtained, it consists of significant ripples. Meanwhile, the line current $i_{\mathrm{s}}$ to the grid also has nontrivial low order ripples at $12.43 \%$ THD as shown in Fig. 5. According to the previous analysis, this comes from both the injection of the LCC converter and the resonance between the filter capacitor and the grid inductive impedance.

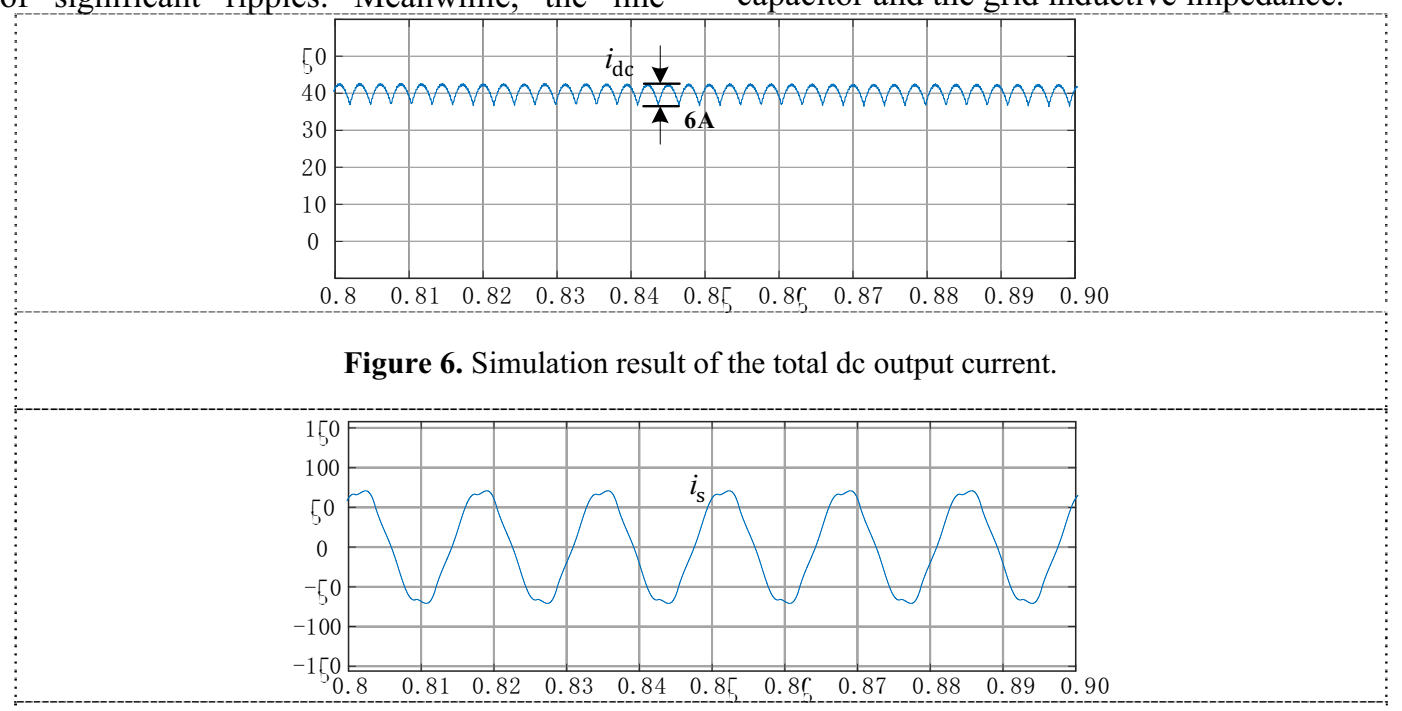

Figure 7. Simulation result of the line current.

Secondly, according to Fig. 6 to Fig. 8, when the CSC converter operates but without using the proposed internal harmonic compensation approach and the virtual impedance based active damping, it is seen that the CSC converter has a good control of its dc rail current that can contribute to the dc de-icing performance. However, it is seen that can ac side grid current still has nontrivial distortions with 5.32\% THD.

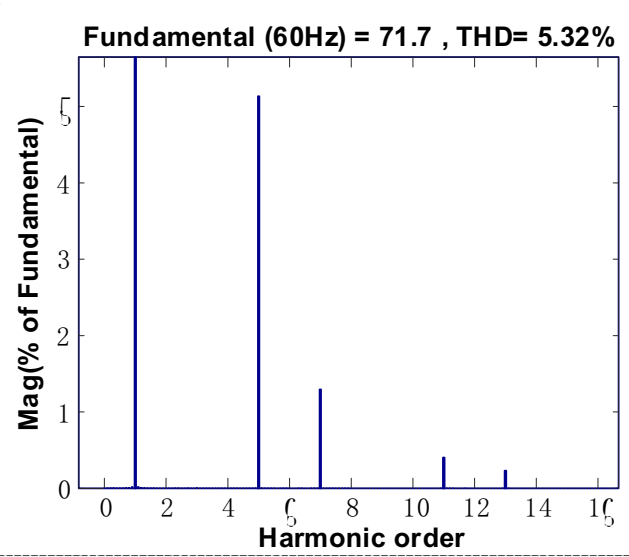

Figure 8. FFT analysis results of the line current. 
Then, when the proposed active damping approach and the active harmonic compensation approach are applied to the system, the corresponding performance is shown in Fig. 9 and Fig. 10. As seen, the line current $i_{\mathrm{s}}$ in this case is quite sinusoidal with $2.30 \%$ THD.
Meanwhile, it is also seen that the total dc output current regulation is accurate at $40 \mathrm{~A}$. Comparing to the counterpart using the conventional approach, it is also seen that the ripples in the dc output current is slightly reduced from $6 \mathrm{~A}$ to $3 \mathrm{~A}$.

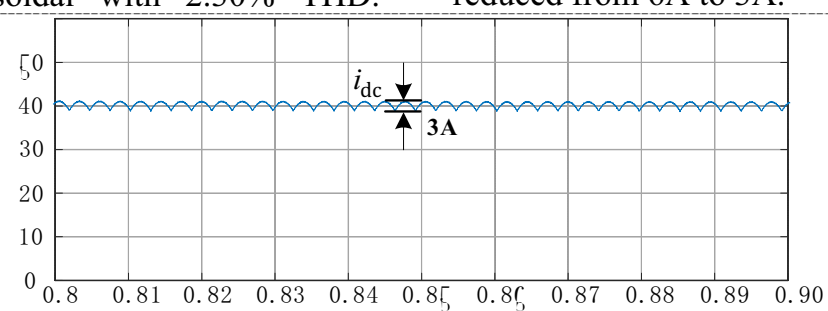

Figure 9. Simulation result of the total dc output current.

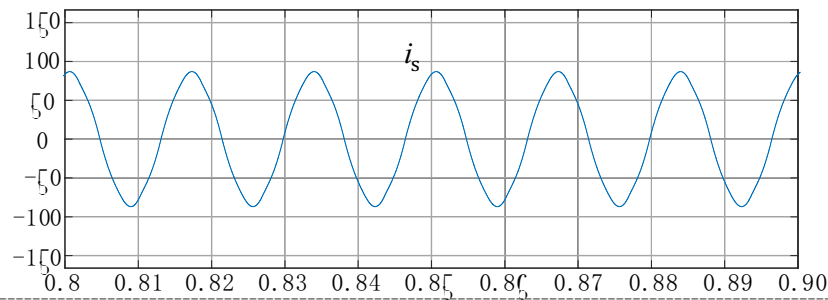

Figure 10. Simulation result of the line current.

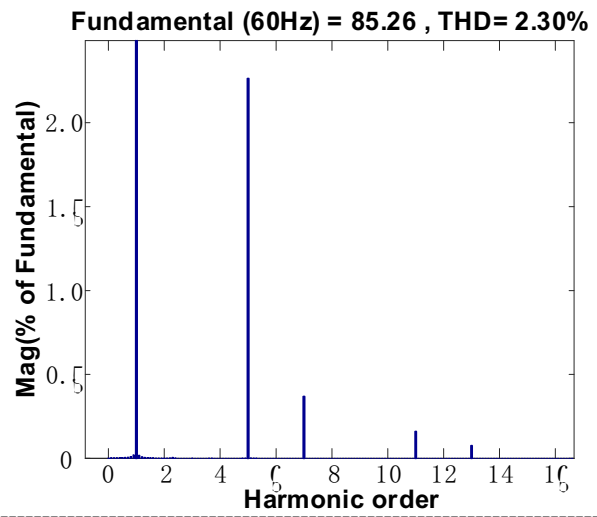

Figure 11. FFT analysis results of the line current.

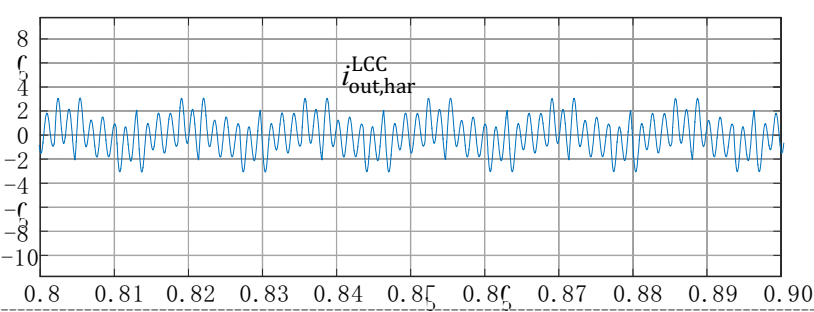

Figure 12. Simulation result of reference current for harmonic compensation.

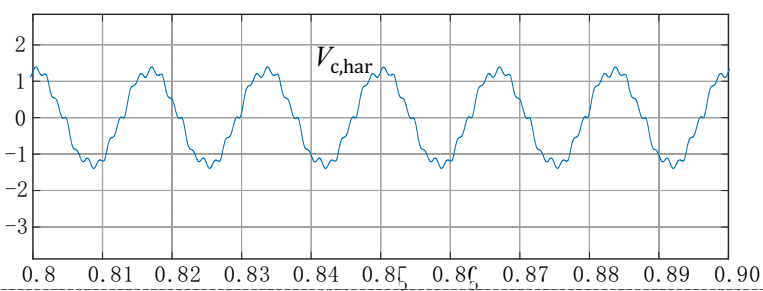

Figure 13. Simulation result of reference current for active damping. 
Finally, the details of reference current for harmonic compensation and active damping are shown in Fig. 12 and Fig. 13. As shown, the CSC has a good tracking of harmonic current for active damping and harmonic compensation by using the direct current modulation approach, which is easier than using the PWM voltage source converters.

\section{Conclusions}

In this paper, a cost effective parallel current source converter has been proposed for transmission line dc deicing. The proposed system consists of a high power LCC converter to provide the dominated dc current and a low power PWM CSC converter is adopted to provide a small part of the dc current. In addition, the virtual impedance and the active harmonic filtering are implemented in the PWM CSC converter in order to obtain filter resonance damping and line current harmonic reduction. It has been demonstrated that the proposed system has an accurate regulation of total dc output current without affecting the quality of ac line current.

\section{References}

1. Koval and A.A. Chowdhury, "An investigation into extremeweather caused transmission line unavailability," in Proc. IEEE Power Eng. Soc. Gen. Meeting, 2005, pp.2425-2428.

2. M.Farzaneh, "Systems for prediction and monitoring of iceshedding, anti-icing and de-icing for overhead lines," CIGRÉ Working Group B2.29, Electrano. 25, pp.51-573, Dec. 2010.

3. Raymundo E. Torres-Olguin; Alejandro Garces; Marta Molinas; Tore Undeland, Integration of Offshore Wind Farm Using a Hybrid HVDC Transmission Composed by the PWM CurrentSource Converter and Line-Commutated Converter, IEEE Transactions on Energy Conversion, 2013, 28(1), pp: 125 - 134

4. M. Steurer, C.A. Luongo, P.R. Ribeiro, S. Eckroad, Interaction Between a Superconducting Coil and the Power Electronics Interface on a 100 MJ SMES System, IEEE Trans. on Applied Superconductivity, 2003, 32(2):1806-1809.

5. 1. R. Espinoza, L. A. Moran and 1. I. Guzman, Multi-Level Three-Phase Current Source Inverter based AC Drive for High Performance Applications, IEEE 36th PESC '05., pp. 2553 2559, June, 2005.

6. J. Y. Bao, W. B. Bao, Z. C. Zhang, A simple current-balancing method for a three-phase 5level current-source inverter [C] Industrial Electronics, IECON' 09, 35th Annual Conference of IEEE. Porto: IEEE, 2009: 104-108.

7. M. P. Aguirre, M. A. Engelhardt, J. M. Bracco, Current balance control in a multilevel current source inverter [C]// 2013 IEEE International
Conference on Industrial Technology (ICIT). Cape Town: IEEE, 2013: 1567-1572.

8. J. R. Espinoza; L. A. Moran; J. I. Guzman, Multi Level ThreePhase Current Source Inverter based AC Drive for High Performance Applications, 2005 IEEE 36th Power Electronics Specialists Conference, 2005, pp: 2553 - 2559

9. L. Jun, K. W. E. Cheng, D. Sutanto, and D.H. Xu, A multimdodule hybrid converter for hightemperature superconducting magnetic energy storage systems (HT-SMES), IEEE Trans. Power Delivery, vol. 20, no. 1, pp. 475-480, Jan. 2005.

10. N. Binesh, B. Wu, 5-level parallel current source inverter for high power application with dc current balance, 2011 IEEE International Electric Machines \& Drive Conference (IEMDC)

11. D. W. Xu, N. R. Zargari, B. Wu, et al, A medium voltage AC drive with parallel current source inverters for high power applications[C], IEEE 36th Power Electronics Specialists Conference, PESC'05, Recife: IEEE, pp. 2277-2283, 2005. 